\title{
Programmable Infusion Pumps in ICUs: An Analysis of Corresponding Adverse Drug Events
}

\author{
Teryl K. Nuckols, MD, MSHS ${ }^{1,2}$, Anthony G. Bower, $P h D^{7}$, Susan M. Paddock, PhD', \\ Lee H. Hilborne, MD, MPH ${ }^{1,3}$, Peggy Wallace, RN, MSN ${ }^{7}$, Jeffrey $M$. Rothschild, $M D, M P H^{4}$, \\ Anne Griffin, RN, $M P H^{7}$, Rollin J. Fairbanks, $M D, M S^{5}$, Beverly Carlson, $M S, R N, C N S^{6}$, \\ Robert J. Panzer, $M D^{7}$, and Robert H. Brook, SCD, MD ${ }^{1,2}$
}

\begin{abstract}
'The RAND Corporation, Santa Monica, CA, USA; ${ }^{2}$ Division of General Internal Medicine and Health Services Research, Department of Medicine, David Geffen School of Medicine at the University of California, Los Angeles, Los Angeles, CA, USA; ${ }^{3}$ Department of Pathology and Laboratory Medicine, David Geffen School of Medicine at the University of California, Los Angeles, Los Angeles, CA, USA; ${ }^{4}$ Division of General Internal Medicine, Brigham and Women's Hospital, Boston, MA, USA; ${ }^{5}$ Department of Emergency Medicine, University of Rochester, Rochester, NY, USA; ${ }^{6}$ Clinical Research Department, Sharp HealthCare, San Diego, CA, USA; ${ }^{7}$ Department of Medicine, University of Rochester, Rochester, NY, USA.
\end{abstract}

BACKGROUND: Patients in intensive care units (ICUs) frequently experience adverse drug events involving intravenous medications (IV-ADEs), which are often preventable.

OBJECTIVES: To determine how frequently preventable IV-ADEs in ICUs match the safety features of a programmable infusion pump with safety software ("smart pump") and to suggest potential improvements in smart-pump design.

DESIGN: Using retrospective medical-record review, we examined preventable IV-ADEs in ICUs before and after 2 hospitals replaced conventional pumps with smart pumps. The smart pumps alerted users when programmed to deliver duplicate infusions or continuousinfusion doses outside hospital-defined ranges.

PARTICIPANTS: 4,604 critically ill adults at 1 academic and 1 nonacademic hospital.

MEASUREMENTS: Preventable IV-ADEs matching smart-pump features and errors involved in preventable IV-ADEs.

RESULTS: Of 100 preventable IV-ADEs identified, 4 involved errors matching smart-pump features. Two occurred before and 2 after smart-pump implementation. Overall, 29\% of preventable IV-ADEs involved overdoses; $37 \%$, failures to monitor for potential problems; and $45 \%$, failures to intervene when problems appeared. Error descriptions suggested that expanding smart pumps' capabilities might enable them to prevent more IV-ADEs.

CONCLUSION: The smart pumps we evaluated are unlikely to reduce preventable IV-ADEs in ICUs because they address only $4 \%$ of them. Expanding smart-pump capabilities might prevent more IV-ADEs.

KEY WORDS: drug therapy; medication error; prevention and control; infusion pump; decision making, computer-assisted; critical care.

J Gen Intern Med 23(Suppl 1):41-5

DOI: $10.1007 / \mathrm{s} 11606-007-0414-y$

(c) Society of General Internal Medicine 2007

\section{INTRODUCTION}

Preventable adverse drug events involving intravenous medications (preventable IV-ADEs: injuries owing to intravenous medication errors) occur frequently in intensive care units (ICUs). ${ }^{1,2}$ Many errors occur during medication administration, and calculating infusion rates and programming pumps are high-risk steps. ${ }^{2,3}$ Programmable infusion pumps with safety software ("smart pumps") were designed to intercept such errors by displaying alerts if continuous-infusion dosages exceed hospital-defined ranges or, for one pump, if duplicate infusions are administered. ${ }^{4}$

Three studies indicate that the reprogramming of smart pumps in response to alerts occurs frequently. ${ }^{5-7}$ However, because most medication errors happen not to cause harm, ${ }^{2,8}$ the ability of smart pumps to reduce IV-ADEs also warrants investigation. One study evaluated whether smart pumps prevent IV-ADEs and found no significant decrease. ${ }^{8}$ No studies have assessed how often preventable IV-ADEs actually match smart-pump safety features or whether expanding pump capabilities might prevent additional types of IV-ADEs.

In this study, we sought to determine how frequently preventable IV-ADEs match one smart pump's features and to identify additional smart-pump capabilities. Our ultimate objective was to compare preventable IV-ADE incidence rates between smart pumps and conventional pumps.

\section{METHODS}

\section{Overview}

We used quantitative and qualitative methods to examine preventable IV-ADEs in adult ICUs before and after 2 hospitals replaced conventional pumps with smart pumps.

\section{Setting and Subjects}

With assistance from the study sponsor (Cardinal Health, San Diego, CA, USA), we selected 2 hospitals that had implemented their smart pumps in 2003 (Alaris version 5): an academic hospital in the northeast and a nonacademic hospital in the west. We examined 20,000 bed-days of care in surgical, 
trauma/burn, and medical ICUs, half during conventionalpump study periods and half during smart-pump periods. We obtained Institutional Review Board approvals; informed consent was not required.

\section{Smart-Pump Features}

A programmable unit within the pump controlled up to 4 attached infusion devices and recorded ("logged") programming errors, alerts, reprogramming events, and overrides. Before implementation, study hospitals developed software libraries for drugs given by continuous infusion; bolus dosing was not addressed. The manufacturer suggested dosing limits then hospitals made modifications. ICU libraries included over 50 drugs

Nurses could program the pumps as if they were conventional or use the library's safety features. Once in the library, nurses selected a drug and standard concentration then entered the dose. For weight-based dosing, nurses entered patient weights and doses, then the software calculated flow rates. When doses exceeded library limits or duplicate infusions were programmed, the software provided alerts and prevented infusion until they were addressed; overrides were permitted.

\section{Data Collection}

This included abstracting medical records and rating suspected ADEs. We did not observe pump use or abstract smartpump logs.

Critical care nurses (4-5 per hospital; uninvolved in study participants' care) received 4-day trainings in medical-record abstraction and $\mathrm{ADE}$ identification (reading manuals, discussing examples, and reviewing sample records). During data collection, nurses recorded clinical and demographic data then used manual trigger-tool review (which uses key words as sentinels to identify $\mathrm{ADEs})^{9}$ plus implicit review (professional judgment) to identify suspected ADEs and any events that infusion pumps might have caused. For each suspected ADE, they summarized relevant details of medication therapy, ${ }^{10}$ errors, and injuries. Reliability assessment involved reabstracting 100 records.

Four board-certified Internal Medicine physicians (unaffiliated with study hospitals) rated suspected ADEs. Two reviewed each electronic summary individually then met to reach consensus, rating: whether an $\mathrm{ADE}$ occurred, ${ }^{2}$ drugs involved, routes/modes of administration, ${ }^{2}$ preventability, ${ }^{11}$ errors involved ${ }^{2}$ and whether errors matched smart-pump functions at each hospital (i.e., whether continuous-infusion doses were outside library limits or present in multiple infusions). Reliability assessment involved randomly selecting 50 suspected ADEs.

\section{Quantitative Analysis}

We used SAS version 9.1.3. First, we examined administration routes/modes, drugs, stages in delivery, and errors involved in preventable IV-ADEs overall. Second, we calculated incidence rates (preventable IV-ADEs per 1,000 patient-days in ICU) for preventable IV-ADEs matching smart-pump functions in each study period. Third, we compared total preventable IV-ADEs incidence rates between conventional-pump and smart-pump periods using Poisson regression models that included multiple demographic and clinical variables. We had $80 \%$ power (alpha $=0.05$ ) to detect a $46 \%$ change. Finally, we determined intracluster correlations for nurse and physician ratings. ${ }^{12}$

\section{Qualitative Analysis}

For events matching smart-pump features, we summarized errors involved. For other events, we summarized examples of common errors and explored additional smart-pump capabilities.

\section{RESULTS}

Among 4,604 patients with 20,559 bed-days in ICU, we identified 100 preventable IV-ADEs (4.86 per 1,000 patientdays). Half of preventable IV-ADEs involved continuous infusions and $40 \%$ boluses. Morphine, insulin, fentanyl, and propofol represented $44 \%$ of all drugs involved. Half of errors occurred during ordering, 14\% during administration, and 35\% during monitoring. Failure to intervene, failure to monitor $37 \%$ combining subtypes, not shown), and overdose were common errors (Table 1). Two preventable IV-ADEs in each study period (4\%, 0.19 per 1,000 patient-days) matched smart-pump functions at each hospital (Table 2). No pumps caused any injuries.

As noted in the Introduction, our ultimate objective was to compare total preventable IV-ADEs incidence rates between conventional-pump and smart-pump periods. However, given that few preventable IV-ADEs matched smart-pump features, statistical power was severely inadequate. The conventionalpump rate was 4.78 per 1,000 patient-days and the smartpump rate was 4.95 (adjusted mean difference 0.04 per 1,000 patient-days, $p=0.96$ ).

Event descriptions suggested that expanding smart-pump library applications, integrating pumps with vital-sign and laboratory data, integrating pumps with computerized physician order entry (CPOE), and automating medication titration

Table 1. Types of IV Medication Errors Causing Preventable IVADEs in Both Study Periods Combined

\begin{tabular}{lr}
\hline \hline Errors & \\
\hline Errors relating to dose of medication received & $29(29.0 \%)$ \\
Improper dose: overdose, no. (\%) & $3(3.0 \%)$ \\
Improper dose: underdose, no. (\%) & $0(0.0 \%)$ \\
Improper dose: extra dose, no. (\%) & $1(1.0 \%)$ \\
Wrong strength/concentration: too high, no. (\%) & $1(1.0 \%)$ \\
Wrong rate, no. (\%) & $4(4.0 \%)$ \\
Wrong duration, no. (\%) & $5(5.0 \%)$ \\
Errors relating to administration details & $5(5.0 \%)$ \\
Wrong technique, no. (\%) & $1(1.0 \%)$ \\
Wrong drug, no. (\%) & $5(5.0 \%)$ \\
Wrong time, no. (\%) & $3(3.0 \%)$ \\
Errors relating to monitoring for potential problems & $1(1.0 \%)$ \\
Failure to monitor: drug-disease interaction, no. (\%) & $9(9.0 \%)$ \\
Failure to monitor: allergy, no. (\%) & $19(19.0 \%)$ \\
Failure to monitor: drug-drug interaction, no. (\%) & \\
Failure to monitor: laboratory, no. (\%) & $45(45.0 \%)$ \\
Failure to monitor: other, no. (\%) & $13(13.0 \%)$ \\
Errors relating to intervening after problems appear & 100 \\
Failure to intervene, no. (\%) & \\
Other errors & \\
Total preventable IV-ADEs* &
\end{tabular}

*Each preventable IV-ADE may be associated with more than 1 type of error.

$I V-A D E=$ adverse drug events involving intravenous medications 
Table 2. Qualitative Analysis of Errors Causing Preventable IV-ADEs

\begin{tabular}{lc}
\hline IV-ADE Description & Description of Smart-pump Features \\
\hline Preventable IV-ADEs matching existing smart-pump functions at the same hospital and existing smart-pump functions
\end{tabular}

Overdose: A middle-aged female with a stroke was prescribed an IV labetalol infusion for blood pressure control. Pharmacy sent 2 medication bags to the unit, 1 containing $1 \mathrm{mg} / \mathrm{mL}$ labetalol and, $2 \mathrm{~h}$ later, another containing $2 \mathrm{mg} / \mathrm{mL}$. The $1-\mathrm{mg} / \mathrm{mL}$ solution infused at $100 \mathrm{mg} / \mathrm{h}$ and the $2 \mathrm{mg} / \mathrm{mL}$ solution infused concurrently in a second line at $140 \mathrm{mg} / \mathrm{h}$. The blood pressure fell to 85/70 then the second infusion was stopped and the blood pressure recovered.

Overdose: A middle-aged male with delerium tremens received a lorazepam infusion at $20 \mathrm{mg} / \mathrm{h}$ and subsequently an IV propofol infusion at $70 \mathrm{mcg} / \mathrm{kg} / \mathrm{min}$. His blood pressure and oxygen saturation declined, the 2 medications were titrated off over the next hour and a half, and then the blood pressure recovered.

Overdose: A middle-aged male with renal failure received increasing doses of an insulin infusion for uncontrolled blood glucose (per protocol), peaking at 24 units/h for blood glucose values in the 400s. Over the next $6 \mathrm{~h}$, the blood glucose declined and the insulin was titrated off, but the blood glucose dropped into the 20s.

Overdose: A young adult male received a propofol infusion for ventilator sedation. It was started at $70 \mathrm{mcg} / \mathrm{kg} / \mathrm{min}$ and increased to $100 \mathrm{mcg} / \mathrm{kg} / \mathrm{min}$ $10 \mathrm{~min}$ later. His blood pressure fell to $70 / 50$, and then recovered after the propofol was stopped.
Practice standardization: After smart pumps implementation, $1 \mathrm{mg} / \mathrm{mL}$ was the only concentration of labetalol used in ICUs at this hospital.

Overdose detection: Smart pumps at this hospital alerted nurses when labetalol infused at a dose above $120 \mathrm{mg} / \mathrm{h}$.

Duplicate medication detection: Smart pumps at this hospital alerted nurses when labetalol was administered concurrently in 2 different lines.

Overdose detection: Smart pumps at this hospital alerted nurses when lorazepam infused at a dose above $10 \mathrm{mg} / \mathrm{h}$ (or propofol at a dose above $100 \mathrm{mcg} / \mathrm{kg} / \mathrm{min})$.

Overdose detection: Smart pumps at this hospital alerted nurses when insulin infused at a dose above than 20 units/h.

Overdose detection: Smart pumps at this hospital alerted nurses when propofol infused at a dose above $80 \mathrm{mcg} / \mathrm{kg} / \mathrm{min}$.

Examples of common preventable IV-ADEs and expanding pump library capabilities to prevent additional IV-ADEs

Failure to intervene: A middle-aged female on morphine PCA pump for postoperative pain experienced 3 episodes of vomiting. She received 3 doses of ondansetron and 1 of promethazine. After $24 \mathrm{~h}$, the opiate was changed to hydromorphone and the vomiting stopped.

Overdose: An elderly female with a stroke and a systolic blood pressure in 190s received 2 doses of IV hydralazine $20 \mathrm{mg} 5 \mathrm{~min}$ apart. She developed hypotension and a severe brain stem infarct.
Modifications to intercept errors: For patients on IVopiates, a smart pump could detect when IV antiemetics are given and alert nurses that switching opiates should be considered.

Modifications to intercept errors: For patients on IV hydralazine, a smart pump could track cumulative doses, have limits per designated period of time, and alert nurses if doses exceeded these limits.

Examples of common preventable IV-ADEs and incorporating real-time vital-sign or laboratory test data to prevent additional IV-ADEs

Overdose, failure to monitor, failure to intervene: An elderly male was given $100 \mathrm{mcg}$ IV fentanyl bolus and propofol infusion at $35 \mathrm{mcg} / \mathrm{kg} / \mathrm{min}$ for a bedside procedure. Blood pressure dropped to 60 s systolic. $\mathrm{He}$ received $250 \mathrm{~mL}$ of normal saline, but propofol was not changed. Blood pressure was rechecked 90 min later and was still in 60s systolic.

Failure to monitor, failure to intervene: An elderly female was started on an IV heparin infusion for acute myocardial infarction. Daily PTT results were repeatedly above therapeutic range. Dose was lowered but PTT was not repeated until the next day when it was still high. Patient developed a retroperitoneal hematoma and died.

Failure to monitor: An elderly female with end-stage renal failure was a given standard insulin infusion protocol to manage her blood glucose but no glucose was provided via the enteral route or IV. Her blood glucose dropped to 33 then rebounded to over 200 after glucose was given.
Modifications to intercept errors: For patients on propofol or other vasoactive IV drugs, a smart pump could receive blood pressure data, and alert nurses or shut off when blood pressure remains outside a defined range for more than a few minutes.

Modifications to automate tasks: For patients on propofol or other vasoactive IV drugs, a smart pump could receive blood pressure data and titrate the drugs to keep blood pressure within a defined range.

Modifications to intercept errors: For patients on IV heparin, a smart pump could receive PTT data and alert nurses if PTT were not checked according to the frequency defined in the protocol or if PTT were above or below desired range.

Modifications to automate tasks: For patients on IV heparin, a smart pump could receive PTT data and titrate heparin according to a defined protocol. A smart pump could interact with computerized physician order entry, which could order PTT to be checked according to the frequency defined in the protocol.

Modifications to intercept errors: For patients on IV insulin, smart pumps could receive blood glucose data, alert nurses when values fall outside of a protocol, check whether protocols are appropriate for renal function, and alert nurses if no IV glucose is provided.

Modifications to automate tasks: For patients on IV insulin, a smart pump could receive blood glucose data and titrate insulin according to a defined protocol.

$I V-A D E=$ adverse drug events involving intravenous medications, $P T T=$ partial thromboplastin time 
might prevent additional IV-ADEs (Table 2). The intracluster correlation for nurses identifying suspected events was 0.39 . Physician intracluster correlations were: for ADEs, 0.71; preventability, 0.90; and smart-pump preventability, 1.0.

\section{DISCUSSION}

Examining preventable IV-ADEs among 4,600 adult ICU patients, we found that only $4 \%$ could be intercepted by a smart pump that detects duplicate and excessive doses of continuous infusions. There were several reasons for the low rate of corresponding events: these pumps did not address boluses, the dosing ranges appeared too wide for certain patients, and monitoring problems were more common than administration ones. Nevertheless, we observed that expanding pump functions and integrating them with real-time clinical data might enable smart-pump technology to reduce other common, harmful errors.

Four evaluations of similar smart pumps came to more positive conclusions. Comparing 426 infused medications with physician orders during a 9-hour period, researchers judged 1 error to be smart-pump-preventable because it was a ratedeviation error involving a pump-programming mistake. ${ }^{13}$ Three studies examining smart-pump logs found that reprogramming events, assumed to be intercepted errors, were common. $^{5-7}$

Because errors have unpredictable relationships to injuries, however, preventable IV-ADEs must also be examined; for example, $0.9 \%$ of medication errors caused ADEs in medical floor units versus $11 \%$ in cardiac ICUs. ${ }^{2,8}$ A controlled trial evaluating a similar pump did consider preventable ADEs and found no significant reduction, because of limited power and because nurses used its safety features inconsistently. ${ }^{8}$

In contrast, our analysis revealed that substantially reducing preventable IV-ADEs in ICUs would require smarter pumps. Many preventable IV-ADEs involved failures to monitor for or adequately respond to undesirable medication effects. Smart-pump technology could alert nurses to such failures by integrating infusions with CPOE, and real-time vital-sign and laboratory data. Perhaps more controversially, smart pumps could automate some titration tasks. Intensive insulin infusions seem particularly amenable to automation because complex protocols create many opportunities for error; clinical assessment is rarely required; and an automated technique for tightly controlling blood glucose, the euglycemic glucose clamp, already exists. ${ }^{14}$ Others have described closedloop systems for titrating sedatives. ${ }^{15}$

The manufacturer of the pumps we evaluated has already expanded applications to include boluses, syringe pumps, and patient-controlled analgesia. An integrated bar-code reader has been designed to address wrong-patient and wrong-drug errors. A patient-controlled analgesia pump monitors oxygen saturation and end-tidal carbon dioxide, issuing an alert or shutting off if values deviate from hospital-defined ranges (Butterfield, personal communication, 2006).

As with any technology, new smart-pump capabilities have potential barriers to overcome. Integration with bar coding and CPOE assumes that hospitals are adopting these technologies, which may not be the case. ${ }^{16}$ Incorporating real-time vital-sign data may require nursing practice changes. Integration with laboratory data would require software development. Auto- mating monitoring and titration tasks would reduce nurses' opportunities to practice these skills. ${ }^{17}$ Finally, new safety technologies can introduce new errors. ${ }^{18}$ Consequently, expanding smart-pump applications will require careful development and testing.

Regarding limitations, we may have underestimated events matching smart-pump functions because we could not evaluate smart-pump logs or observe users. Nursing agreement was only fair, possibly owing to insufficient training or reabstracting too few records; other studies have not reported nurse agreement. However, we used standard methods and documented physician agreement comparable to prior studies. ${ }^{19}$ We studied 2 hospitals, 1 clinical setting, and 1 time period. Smart-pump technology is evolving rapidly and future research should include nonICU settings, where IV-ADEs may not be detected quickly.

\section{CONCLUSIONS}

The smart pumps we evaluated are unlikely to reduce preventable IV-ADEs in ICUs because they address only $4 \%$ of them. Expanding smart-pump capabilities might prevent more IV-ADEs.

Acknowledgements: We thank the 9 nurse abstractors who participated in data collection, administrators at the study hospitals, physicians who helped rate suspected events, and RAND's communications analysts. Funding source: Cardinal Health (formerly Alaris Medical Systems), 10221 Wateridge Circle, San Diego, CA 92121-2772. Representatives of Cardinal Health contributed to and provided feedback on the study design and data collection methods, provided a list of hospitals that had recently adopted Alaris version 5 pumps, and commented on manuscript drafts. RAND staff chose the study design and data collection methods, selected study hospitals, were solely responsible for all aspects of data collection and analysis, and wrote the manuscript. Under the terms of the contract between RAND and Cardinal Health, RAND staff retained complete control over all contents of the manuscript, with leeway to accept or reject any modifications suggested by Cardinal Health representatives. RAND staff strove to be as objective as possible throughout this evaluation. RAND provided some internal support for the preparation of this manuscript. The oral abstract was presented at the Society for General Internal Medicine Annual Meeting, 2006.

Conflict of Interest: Funding for this project was provided to RAND by Cardinal Health (formerly Alaris Medical Systems). Jeffrey M. Rothschild consulted for Aptima, Inc: 2004-2005. The other authors reported no conflicts of interest.

Corresponding Author: Teryl K. Nuckols, MD, MSHS; The RAND Corporation, 1776 Main Street, Box 2138, Santa Monica, CA 904072138, USA (e-mail: teryl@rand.org).

\section{REFERENCES}

1. Rothschild JM, Landrigan CP, Cronin JW, et al. The critical care safety study: The incidence and nature of adverse events and serious medical errors in intensive care. Crit Care Med. 2005;33(8):1694-700.

2. Bates DW, Cullen DJ, Laird N, et al. Incidence of adverse drug events and potential adverse drug events: implications for prevention. JAMA. 1995;274(1):29-34.

3. Apkon M, Leonard J, Probst L, DeLizio L, Vitale R. Design of a safer approach to intravenous drug infusions: failure mode effects analysis. Qual Saf Health Care. 2004;13(4):265-71.

4. Alaris Medical Systems. Guardrails ${ }^{\circledR}$ suite of safety software for the Alaris system. Available at: http://www.cardinalhealth.com/alaris/pro ducts/Guardrails/. Accessed September 27, 2007. 
5. Fields M, Peterman J. Intravenous medication safety system averts high-risk medication errors and provides actionable data. Nurs Adm $\mathrm{B}$. 2005;29(1):78-87.

6. Hatcher I, Sullivan M, Hutchinson J, Thurman S, Gaffney FA. An intravenous medication safety system: preventing high-risk medication errors at the point of care. J Nurs Adm. 2004;34(10):437-9.

7. Malashock CM, Shull SS, Gould DA. Effect of smart infusion pumps on medication errors related to infusion device programming. Hosp Pharm. 2004;39(5):460-9.

8. Rothschild JM, Keohane CA, Cook EF, et al. A controlled trial of smart infusion pumps to improve medication safety in critically ill patients. Crit Care Med. 2005;33(3):533-40.

9. Rozich JD, Haraden CR, Resar RK. Adverse drug event trigger tool: a practical methodology for measuring medication related harm. Qual Saf Health Care. 2003;12(3):194-200.

10. Naranjo CA, Busto U, Sellers EM, et al. A method for estimating the probability of adverse drug reactions. Clin Pharmacol Ther. 1981;30 (2):239-45

11. Dubois RW, Brook RH. Preventable deaths: who, how often, and why? Ann Intern Med. 1988;109(7):582-9.
12. Landis RJ, Koch GG. A one-way components of variance model for categorical data. Biometrics. 1977;33(4):671-9.

13. Husch M, Sullivan C, Rooney D, et al. Insights from the sharp end of intravenous medication errors: implications for infusion pump technology. Qual Saf Health Care. 2005;14(2):80-6.

14. Heinemann L, Anderson JH Jr. Measurement of insulin absorption and insulin action. Diabetes Technol Ther. 2004;6(5):698-718.

15. Gentilini A, Schaniel C, Morari M, Bieniok C, Wymann R, Schnider T. A new paradigm for the closed-loop intraoperative administration of analgesics in humans. IEEE Trans Biomed Eng. 2002;49(4):289-99.

16. Poon EG, Blumenthal D, Jaggi T, Honour MM, Bates DW, Kaushal R. Overcoming barriers to adopting and implementing computerized physician order entry systems in U.S. hospitals. Health Aff (Millwood). 2004;23(4): 184-90.

17. Wickens CD, Lee J, Liu Y, Becker SG. An Introduction to Human Factors Engineering. Saddle, NJ: Pearson/Prentice Hall; 2004:426.

18. Koppel R, Metlay JP, Cohen A, et al. Role of computerized physician order entry systems in facilitating medication errors. JAMA. 2005;293(10):1197-203.

19. Morimoto T, Gandhi TK, Seger AC, Hsieh TC, Bates DW. Adverse drug events and medication errors: detection and classification methods. Qual Saf Health Care. 2004;13(4):306-14. 